\title{
Baby MIND: A magnetised spectrometer for the WAGASCI experiment
}

\author{
Sven-Patrik Hallsjö* \\ University of Glasgow, School of Physics and Astronomy, Glasgow, UK \\ E-mail: p.hallsjo.1@research.gla.ac.uk
}

\section{on behalf of the Baby MIND Collaboration}

The WAGASCI experiment being built at the J-PARC neutrino beam line will measure the ratio of cross sections from neutrinos interacting with a water and scintillator targets, in order to constrain neutrino cross sections, essential for the T2K neutrino oscillation measurements. A prototype Magnetised Iron Neutrino Detector (MIND), called Baby MIND, has been constructed at CERN and will act as a magnetic spectrometer behind the main WAGASCI target. Baby MIND will be installed inside the WAGASCI cavern at J-PARC in the beginning of 2018. Baby MIND will be able to measure the charge and momentum of the outgoing muon from neutrino charged current interactions, to enable full neutrino event reconstruction in WAGASCI.

During the summer of 2017, Baby MIND was operated and characterised at the T9 test beam at CERN. Results from this test beam will be presented, including charge identification performance and momentum resolution for charged tracks. These results will be compared to the Monte Carlo simulations. Finally, simulations of charge-current quasi-elastic (CCQE) neutrino interactions in an active scintillator neutrino target, followed by the Baby MIND spectrometer, will be shown to demonstrate the capability of this detector set-up to perform cross-section measurements under different assumptions.

The 19th International Workshop on Neutrinos from Accelerators-NUFACT2017

25-30 September, 2017

Uppsala University, Uppsala, Sweden

\footnotetext{
* Speaker.
} 


\section{Motivation}

Magnetised Iron Neutrino Detectors (MINDs) have been proposed for Neutrino Factories, since charge reconstruction is required to distinguish $\overline{v_{\mu}}$ appearance and $v_{\mu}$ disappearance in a $v_{\mu}$ and $\bar{v}_{e}$ beam. Baby MIND is a prototype detector to study charge identification of muons on a charged particle beamline at CERN. The CERN Neutrino Platform approved Baby MIND as experiment NP05 in December 2015 and construction started in August 2016 and finished in June 2017. During the development of the detector it was proposed to use Baby MIND as a muon spectrometer downstream of the WAGASCI experiment (T59) at J-PARC, using neutrinos from the T2K beamline, to provide charge and momentum of outgoing muons from neutrino charged current interactions. Currently the schedule is to install Baby MIND in the ND280 pit at the start of 2018.

\section{Design}

Compared to previous magnetised iron detectors, such as CDHS [1] and MINOS [2], both in the kiloton range, Baby MIND is comparatively small, only $65 \mathrm{t}$, and has a fully modular design with separate scintillator modules and magnetic iron modules [3]. These can be set in any configuration as long as a support frame can be built. The use of magnet modules, instead of requiring the use of an all encompassing magnet, simplifies the magnetic design, lowers the cost and allows for a more uniform field.

The layout, seen in figure 1, shows that the full detector is around 4 metres long and is composed of 18 scintillator modules and 33 magnet modules. The detector is divided into four blocks. The gaps between sub-blocks in block 1 have been added to improve the low momentum reconstruction using a lever-arm approach.
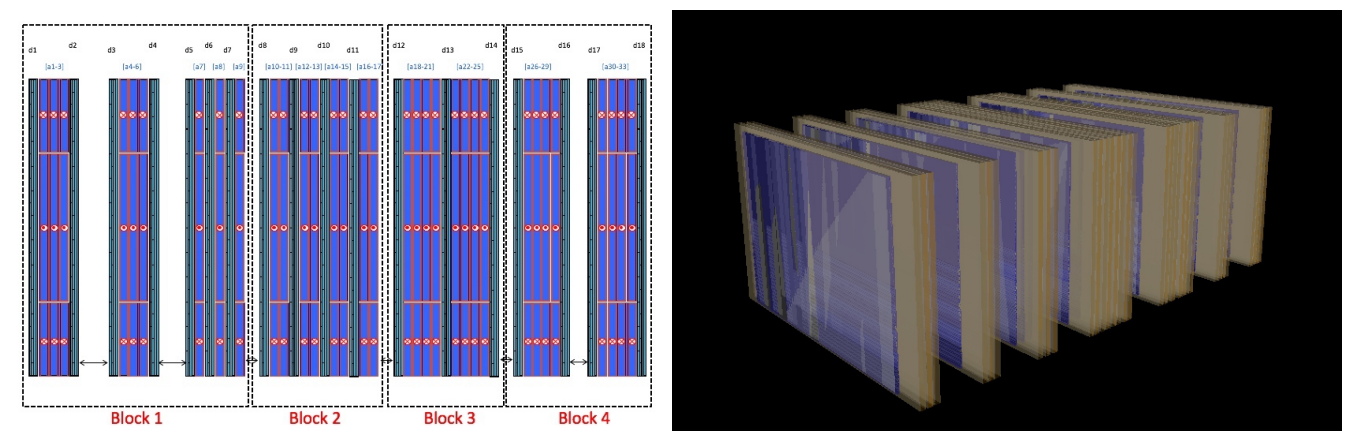

Figure 1: Layout of the Baby MIND.

\subsection{Magnetisation}

The magnet design consists of individually magnetised iron (ARMCO steel) plates denoted as the magnet modules. Each module consists of ARMCO steel with two slits to allow aluminium coils to be wrapped around the steel. The field is split into 3 parts where the field is $1.5 \mathrm{~T}$ but with opposite orientation as can be seen in the figure 2 (right). Because of this simple design, the field lines are contained in the steel and have negligible stray fields of less than $15 \mathrm{mT}$, with a good uniformity in the area of interest. This provides a bending direction either up or down depending 
on where the particle passes and its charge. The magnet module dimensions $3500 \times 2000 \times 30$ $\mathrm{mm}^{3}(\mathrm{Fe})$, with the field oriented transverse to the beam and bending with respect to the bend in the downstream direction. The magnet modules were constructed at CERN through the CERN Neutrino Platform [4].
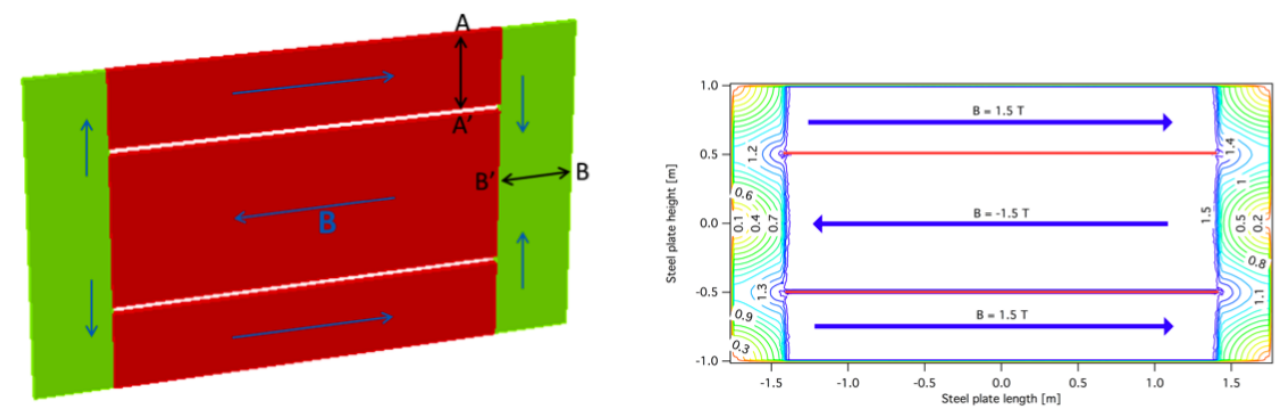

Figure 2: (Left) Schematic view of the magnet module. (Right) A contour plot of the magnet module, with the fiducial areas of interest showing magnetic field uniformity.

\subsection{Scintillator module}

Scintillator bars are used for particle detection to provide both horizontal and vertical position information. Since the vertical information is important for curvature, smaller bars are used to provide a better position resolution. INR Moscow built and designed the scintillator bars, providing a good light yield, regardless of where the bar is hit. Each scintillator module is comprised of 95 horizontal bars, $3000 \times 31 \times 7.5 \mathrm{~mm}^{3}$, and 8 vertical bars, $1950 \times 210 \times 7.5 \mathrm{~mm}^{3}$. The bars are split into four overlapping layers to ensure a $100 \%$ hit efficiency for minimum ionising muons. The bars are polystyrene based, $1.5 \%$ PTP, $0.01 \%$ POPOP and held together mechanically within an aluminium support frame. The total size of the scintillator module is $3000 \times 1950 \times 30 \mathrm{~mm}^{3}$. The bars contain Kuraray WLS fibers (200 ppm, S-type, diameter $1.0 \mathrm{~mm}$ ) and contain a reflective coating 30 to $100 \mu \mathrm{m}$ from chemical etching of the surface. The connectors are custom made using Eljen EJ-500 optical cement.

\subsection{Connectivity}

Hamamatsu MPPC (Multi Pixel Photon Counters) are used to read out the scintillator bars. Compared to PMTs these use a lower voltage, less current and are compatible with magnetic fields. This provides a small size, and simple electronics for the modules. The MPPCs are custom made S12571-025C (and derived S10943-5796), have a size of $1 \times 1 \mathrm{~mm}^{2}$ (65\% fill factor) and $25 \mu \mathrm{m}$ cell size. The operating voltage is $\approx 67.5 \mathrm{~V}$ with photon detection efficiency (PDE) $\approx 35 \%$, gain $5 \times 10^{5}$ and dark counts of typically $100 \mathrm{kcps}$.

\subsection{Electronics}

The MPPC signals, sampled at $400 \mathrm{MHz}$, are powered (HV/LV) and read out by custom made Front End Boards (FEBs) designed for 96 channels using CITIROC [5] ASICs. These rackmounted FEBs have been designed by Geneva University containing $3 \times 32$ channel connectors, 3 


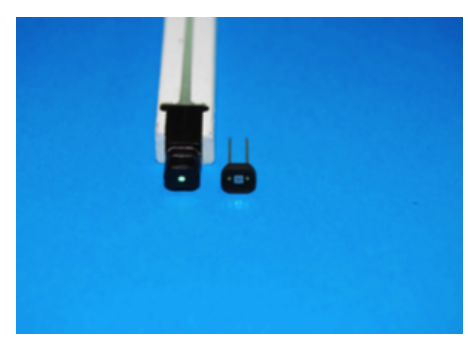

Figure 3: Hamamatsu MPPC with a scintillator bar and connector.
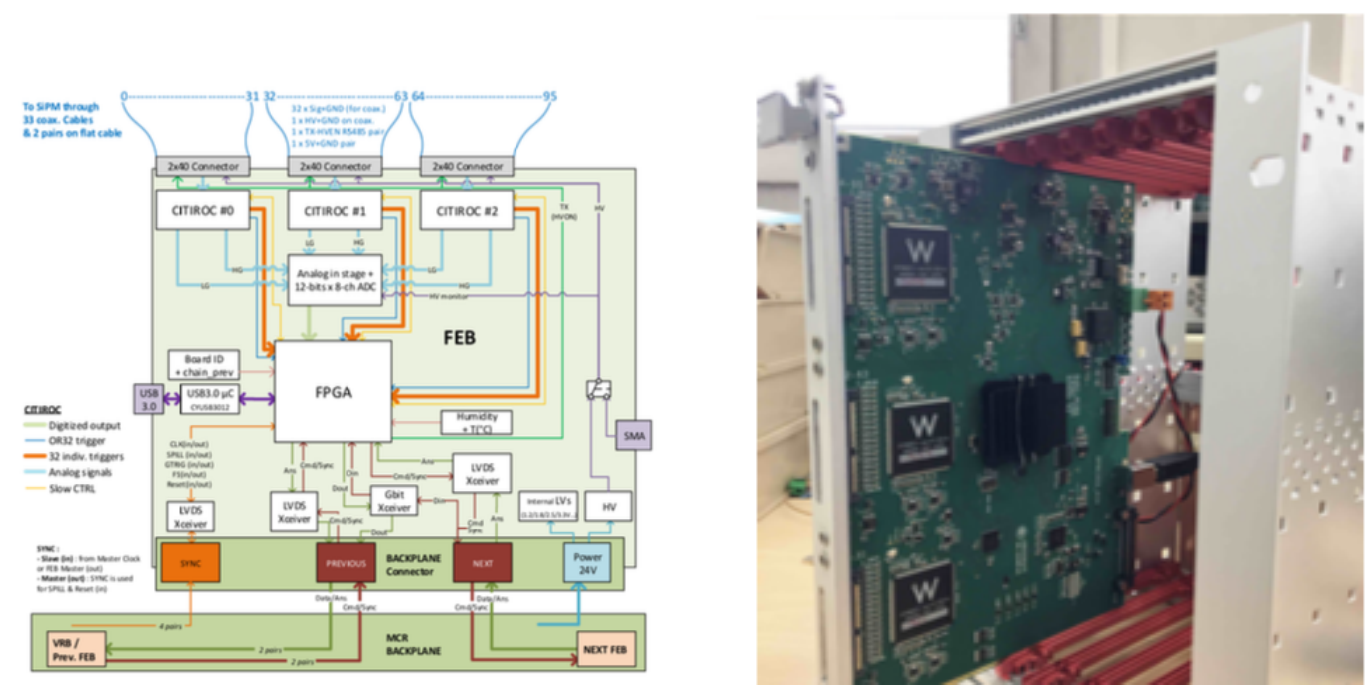

Figure 4: (Left) Schematic view of FEB layout. (Right) An image of the FEB in one of the racks.

CITIROC ASICs with 32 channels each. The FEBs read out and control using a USB3 connector, with Gigabit speed on the backplane on a platform independent Windows/Linux system. There is also an analog readout, $8 \mu$ s for 96-channel low gain and high gain with a 12-bits, 8 channel, 40 MSamples/s per channel ADC based on the Altera ARIA5 FPGA.

\section{Software environment}

The software environment used for Baby MIND is the SaRoMaN (Simulation and Reconstruction of Muons and Neutrinos) software suite [6], developed at the University of Glasgow as a comprehensive software for MIND/nuSTORM (neutrinos from STORed Muons) [7]. The software is accessible on request from https://lspace.ppe.gla.ac.uk.

\section{Test beam}

Baby MIND was exposed to the T9 test beam in 2016 to perform an electronics validation using a TASD (Totally Active Scintillating Detector) [6] and in 2017 to commission with the full Baby MIND. The T9 test beam is derived from the PS synchrotron at CERN, and produces both hadron (pion) and muon beams between 0.5 and $10 \mathrm{GeV} / \mathrm{c}$. 


\subsection{Results}

Sample test beam reconstructed events can be seen in figure 5. For reconstructed simulated events the muon charge efficiency is above $80 \%$ above $500 \mathrm{MeV} / \mathrm{c}$ and above $95 \%$ at $800 \mathrm{MeV} / \mathrm{c}$. Figure 6 shows simulated events and test beam data points. The blue data points are the analysed beam test data at $-2,-3$ and $-5 \mathrm{GeV} / \mathrm{c}$. Simulations and data agree very well. The plots also show the residuals in momentum. The large RMS comes from the RecPack Kalman filter and the simulation bias comes from always underestimating the curvature and thus the momentum. The mismatch between simulation and data may arise from lack of knowledge in the test beam original momentum. The momentum is taken as the value set for the beam magnets, however this may be the momentum of pions before decaying to muons. Full beam line simulations are required to ensure that this is the case.
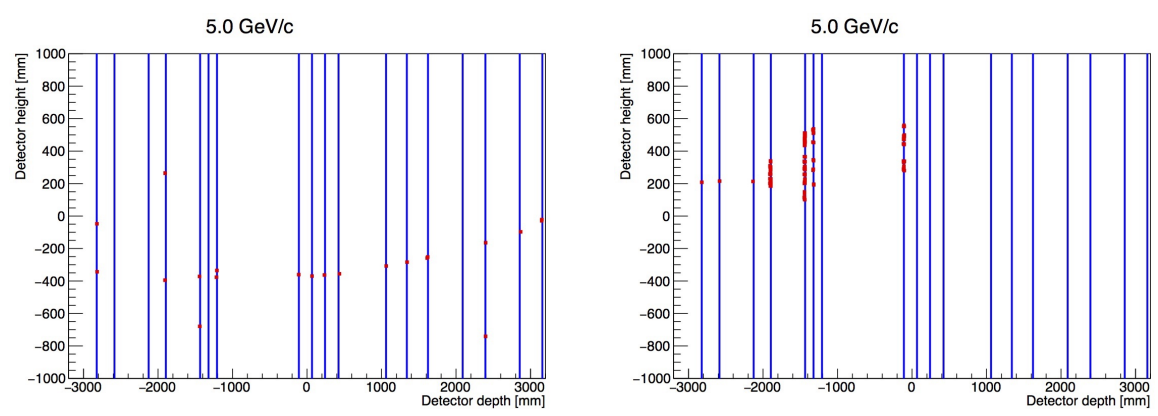

Figure 5: (Left) Example of a $5 \mathrm{GeV} / \mathrm{c}$ muon-like event in Baby MIND. (Right) Example of a $5 \mathrm{GeV} / \mathrm{c}$ pion-like event in Baby MIND.

\section{CCQE studies in a neutrino detector with Baby MIND}

There is an interest to look at charged-current quasi-elastic (CCQE) interactions at energies below $1 \mathrm{GeV} / \mathrm{c}$. Usually iron detectors are not able to handle neutrino interactions at such low energies, however Baby MIND is capable of reconstructing events below $1 \mathrm{GeV} / \mathrm{c}$. Simulations have been carried out with a Totally Active Scintillating Detector (TASD), $1 \times 1 \times 1 \mathrm{~m}^{3}$ with $84 \times 84$ planes, as a target for neutrino interactions with Baby MIND as a spectrometer. A muon neutrino CCQE event has a two-track signature with a muon and a proton, $v_{\mu}+n \rightarrow \mu^{-}+p$, in the TASD, where it is reconstructible if the muon track passes into the Baby MIND. The momentum and charge is then reconstructed in Baby MIND. The simulations have been performed using the nuSTORM [7] neutrino energy spectrum, as seen in figure 7. The neutrino energy reconstruction utilized the following formula,

$$
E_{\text {rec }}=\frac{m_{n} E_{\mu}-m_{\mu}^{2} / 2}{m_{n}-E_{\mu}+\left|\overrightarrow{p_{\mu}}\right| \cos \theta_{\mu}}
$$

where $m_{n}$, is the neutron mass, $m_{\mu}, E_{\mu}, \overrightarrow{p_{\mu}}$, muon mass, energy and three-momentum, and $\theta_{\mu}$ is the scattering angle with respect to the neutrino beam direction. The result of this can be seen in figure 8 , where the missing reconstructed momentum is still under investigation. 

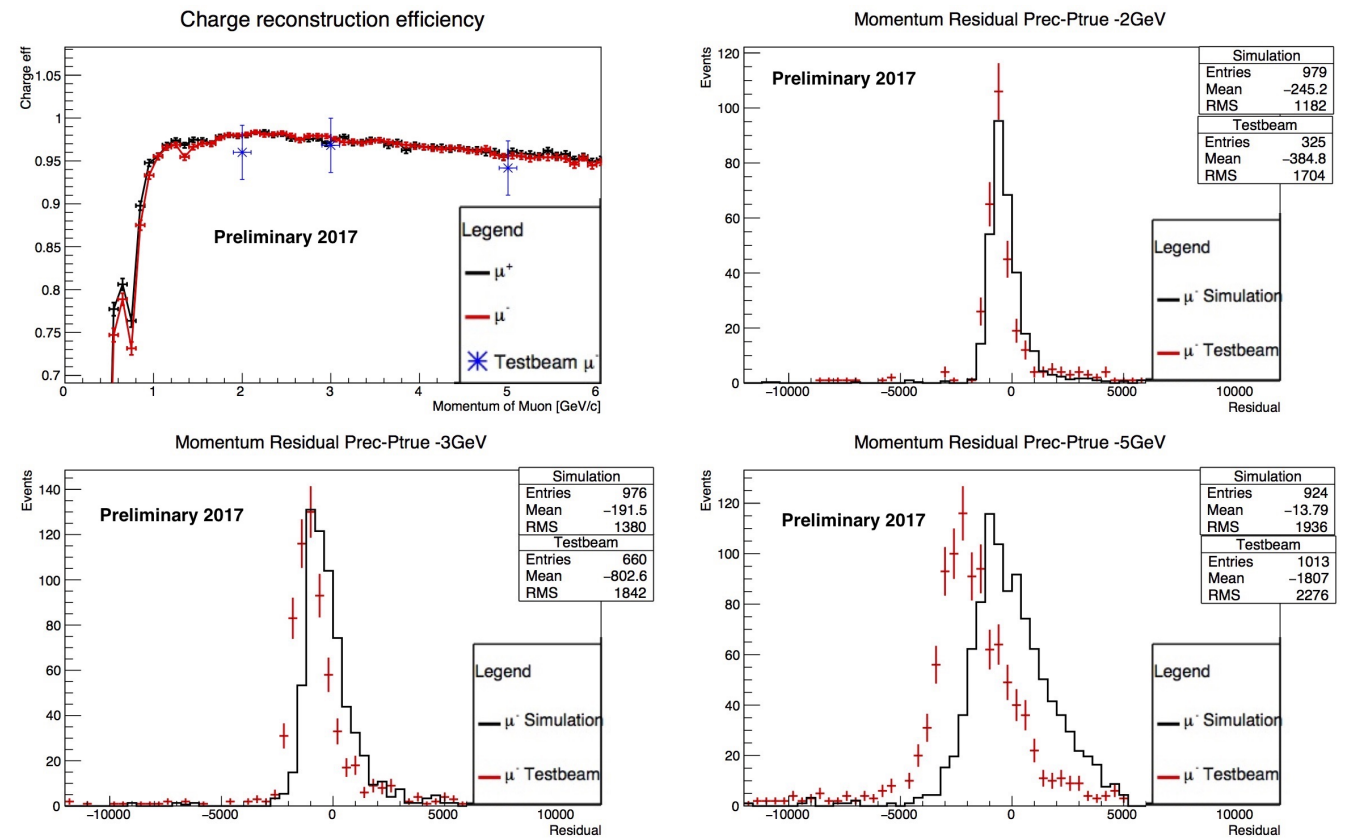

Figure 6: (Top left) Charge identification efficiency for simulated and reconstructed $\mu^{+}$(black) and $\mu^{-}$ (red), and test beam data (blue points). The remaining plots include residual reconstructed momenta in $\mathrm{MeV} / \mathrm{c}$ for simulations (black) and beam test data (red) for $-2,-3$ and $-5 \mathrm{GeV} / \mathrm{c}$ muons.
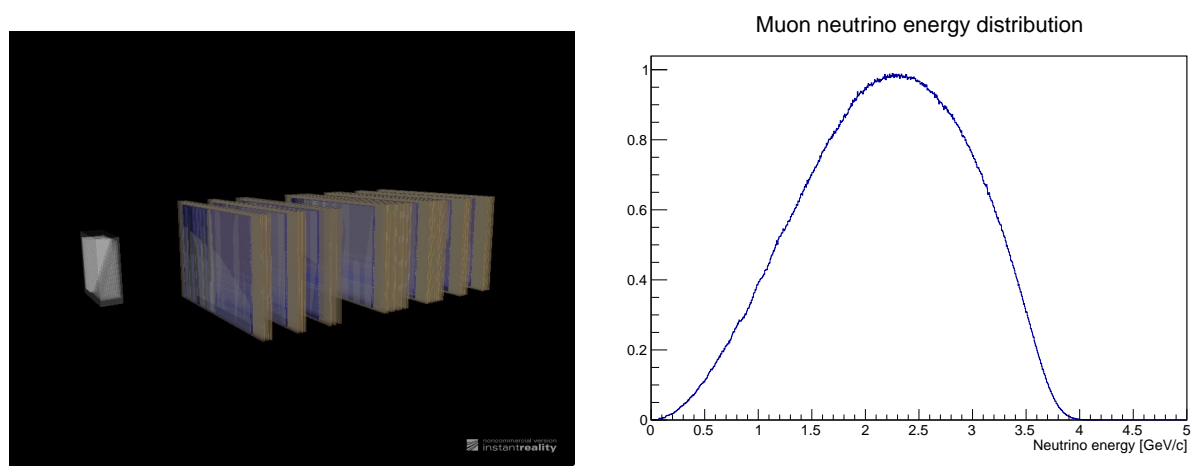

Figure 7: (Left) Schematic view of TASD + MIND. (Right) nuSTORM [7] energy spectrum for muonneutrinos.

\section{Conclusion}

In conclusion, Baby MIND contains magnet modules with a novel design and an innovative magnetisation scheme with optimal flux return that enables far greater flexibility in detector layout compared with previous MIND designs. All 18 scintillator modules have been extensively tested and qualified in a test beam at CERN. Previously MINDs have not been considered to reconstruct articles with momenta lower than $1 \mathrm{GeV} / \mathrm{c}$, however Baby MIND can go down to lower momenta, $\approx 300 \mathrm{MeV} / \mathrm{c}$. Baby MIND can be used both as a standalone muon spectrometer, or as a neutrino detector in combination with other active targets. The next step is the installation of the Baby MIND at the J-PARC ND280 pit in early 2018. 


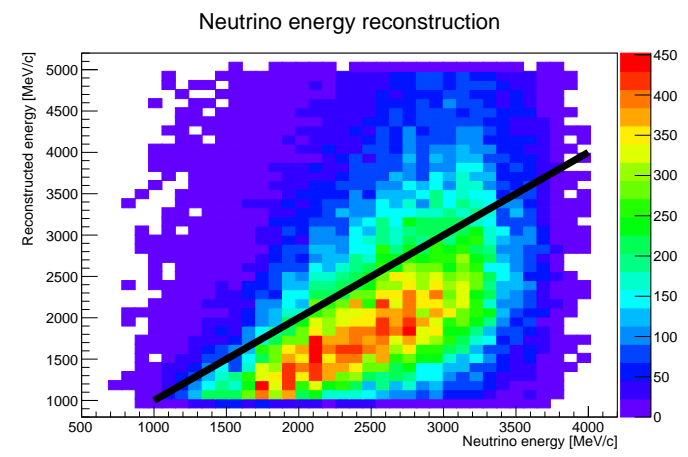

Figure 8: Preliminary $v_{\mu} \mathrm{CCQE}$ energy reconstruction based on simulations, with a black line to emphasise the expected energy.

\section{Acknowledgement}

This project has recived funding from the European Union's Horizon 2020 Research and Innovation programme under Grant Agreement no. 654168

The CERN Neutrino Platform provided extensive support for the design, construction and testing of the Baby MIND. We acknowledge the large contribution made by CERN through the Neutrino Platform to Baby MIND.

\section{References}

[1] F. Dydak et al., A search for $v_{\mu}$ oscillations in the $\Delta m^{2}$ range 0.3-90 eV $V^{2}$, Physics Letters $B$, vol. 134, no. 3, pp 281-286, 1984

[2] A. Habig et al. [MINOS Collaboration], The MINOS detectors, 29th International Cosmic Ray Conference (ICRC 2005) Pune, India, August 3-11, 2005, hep-ex/0507018

[3] M. Antonova et al., The Baby MIND spectrometer for the J-PARC T59(WAGASCI) experiment, PoS(EPS-HEP2017)508

[4] G. Rolando and P. Benoit and A. Blondel and A. Dudarev and E. Noah and H. Pais Da Silva and M. Rayner and H. H. J. ten Kate, New and Optimized Magnetization Scheme for the Baby Magnetized Iron Neutrino Detector at J-PARC, IEEE Transactions on Magnetics 5(53):1-6, 2017)

[5] J. Fleury et al., EASIROC, an easy and versatile readout device for SiPM, Phys. Procedia, vol. 37, pp 1569,2012

[6] M. Antonova et al., Baby MIND: A magnetised spectrometer for the WAGASCI experiment, Proceedings, Prospects in Neutrino Physics (NuPhys2016) : London, UK, December 12-14, 2016, arXiv:1704.08079 [physics.ins-det]

[7] P. Kyberd et al. [nuSTORM Collaboration], arXiv:1206.0294 [hep-ex] 